\title{
IDENTIFICAÇÃO PRÉ-COLHEITA DO RISCO DE OCORRÊNCIA DE “BITTER PIT” EM MAÇÃS 'GALA' POR MEIO DE INFILTRAÇÃO COM MAGNÉSIO E ANÁLISE DOS TEORES DE CÁLCIO E NITROGÊNIO NOS FRUTOS ${ }^{1}$
}

\author{
CASSANDRO VIDAL TALAMINI DO AMARANTE², CRISTIANO ANDRÉ STEFFENS ${ }^{3}$ \\ PAULO ROBERTO ERNANI ${ }^{4}$
}

RESUMO - O "bitter pit" é um distúrbio fisiológico pós-colheita em maçãs, ocasionado pela deficiência de $\mathrm{Ca}$ e agravado pela presença de elevados níveis de $\mathrm{Mg}, \mathrm{N}$ e $\mathrm{K}$ nos frutos. O objetivo deste trabalho foi avaliar a viabilidade prática da infiltração de maçãs 'Gala' com Mg, visando a avaliar, em pré-colheita, o risco de ocorrência de "bitter pit" durante o armazenamento refrigerado, bem como a identificar os atributos minerais do fruto associados à ocorrência do distúrbio. Em 50 talhões de pomares localizados no município de Fraiburgo-SC, foram coletadas amostras de 25 frutos / talhão, cerca de 20 dias antes do início da colheita comercial, sendo os mesmos infiltrados a vácuo com Mg e avaliados quanto à incidência (\%) e severidade (manchas / fruto) de "bitter pit". Nos mesmos talhões, na maturação comercial, foram coletadas amostras de 120 frutos / talhão, sendo que 100 frutos foram armazenados em câmara fria convencional durante quatro meses $\left(0 \pm 0,5^{\circ} \mathrm{C}\right.$ e $90-95 \%$ UR), e 20 frutos foram utilizados para a análise mineral (teores de $\mathrm{Ca}, \mathrm{Mg}, \mathrm{K} \mathrm{e}$ N). Cinco dias após a remoção da câmara fria, os frutos foram avaliados quanto à incidência (\%) e severidade (manchas / fruto) de "bitter pit". Houve correlação linear altamente significativa $\left(\mathrm{r}^{2}=0,69 ; \mathrm{p}<0,001\right)$ entre incidência de "bitter pit" avaliada em frutos infiltrados com Mg e em frutos armazenados em câmara fria. Tanto em maçãs infiltradas com $\mathrm{Mg}$, como naquelas que desenvolveram distúrbio durante o armazenamento em câmara fria, foram observados menores teores de Ca e maiores teores de $\mathrm{N}$ em frutos com elevados níveis de incidência e severidade de "bitter pit". Os resultados obtidos mostram que a infiltração pré-colheita de maçãs 'Gala' com Mg é um método viável visando a identificar talhões em um pomar comercial, quanto ao risco de ocorrência de "bitter pit" durante armazenamento refrigerado.

Termos de indexação: Malus domestica Borkh., composição mineral, distúrbio fisiológico, análise multivariada.

\section{PREHARVEST IDENTIFICATION OF BITTER PIT RISK IN ‘GALA’APPLES BY FRUIT INFILTRATION WITH MAGNESIUM AND ANALYSIS OF FRUIT CONTENTS OF CALCIUM AND NITROGEN}

\begin{abstract}
Bitter pit is a postharvest physiological disorder in apples, related to Ca deficiency, and aggravated by high levels of $\mathrm{Mg}, \mathrm{N}$, and $\mathrm{K}$ in the fruits. This work was carried out to assess the practical viability of 'Gala' apples infiltration with $\mathrm{Mg}$, for preharvest identification of bitter pit risk during cold storage, as well as, to identify the mineral attributes associated with the occurrence of the disorder. Fruits were sampled in 50 plots of apple orchards located in Fraiburgo, SC (Southern of Brazil). Samples of 25 fruits / plot were harvested about 20 days before commercial harvesting, and then vacuum infiltrated with $\mathrm{Mg}$ and assessed for incidence (\%) and severity (pits / fruit) of bitter pit. Samples of 120 fruits were harvested in the same plots, at the commercial maturity. One hundred fruits were stored in conventional cold storage for four months $\left(0 \pm 0.5^{\circ} \mathrm{C}\right.$ and $\left.90-95 \% \mathrm{RH}\right)$ and 20 fruits were analyzed for mineral content $(\mathrm{Ca}, \mathrm{Mg}, \mathrm{K}$, and $\mathrm{N})$. Cold stored fruit were assessed for incidence (\%) and severity (pits / fruit) of bitter pit five days after removal from storage. There was a highly significant linear correlation $\left(r^{2}=0.69 ; p<0.001\right)$ between the incidence of bitter pit assessed in fruits infiltrated with $\mathrm{Mg}$ and in fruits left in cold storage. In both, fruits infiltrated with $\mathrm{Mg}$ and fruits left in cold storage, there was a decrease in Ca and an increase in $\mathrm{N}$ contents with increases of incidence and severity of bitter pit. Results show that preharvest infiltration of 'Gala' apples with $\mathrm{Mg}$ is a viable method to identified plots in a commercial orchard with risk to manifest bitter pit during cold storage.
\end{abstract}

Index terms: Malus domestica Borkh., mineral composition, physiological disorder, multivariate analysis.

1(Trabalho 033-09). Recebido em: 22-01-2009. Aceito para publicação em: 18-09-2009.

${ }^{2}$ Ph.D., bolsista de produtividade em pesquisa do CNPq. Prof. do Depto. de Agronomia, CAV/UDESC,. Cx. P. 281, CEP 88520-000, Lages- SC. Autor para correspondência. E-mail: amarante@cav.udesc.br

${ }^{3}$ Dr., Professor do Departamento de Agronomia, CAV/UDESC, Lages-SC. E-mail: steffens@cav.udesc.br

${ }^{4}$ Ph.D., bolsista de produtividade em pesquisa do CNPq. Prof. do Depto. de Solos e Recursos Naturais, CAV/UDESC, Lages-SC. Email: a2pre@cav.udesc.br 


\section{INTRODUÇÃO}

O "bitter pit" é uma desordem fisiológica que pode ocasionar grandes perdas na cultura da macieira (Ferguson \& Watkins, 1989). Este distúrbio é caracterizado pelo colapso de células da polpa abaixo da casca, criando pequenas depressões de cor escura na superfície do fruto, principalmente na porção distal (Drazěta et al., 2004; Saure, 2005). O "bitter pit" geralmente se desenvolve durante a fase de frigoconservação. Porém, em casos severos, na ausência de medidas de prevenção ou em anos com verão extremamente quente, este distúrbio pode ocorrer ainda na planta (Ferguson \& Watkins, 1989). No Brasil, este distúrbio ocorre principalmente em maçãs 'Gala', 'Fuji', 'Golden Delicious', 'Braeburn' e 'Catarina' (Argenta \& Suzuki, 1994; Nachtigall \& Freire, 1998; Amarante et al., 2005; Amarante et al., 2006a; Amarante et al., 2006b).

$\mathrm{O}$ "bitter pit" ocorre em frutos que apresentam baixos teores de Ca, e é agravado pela existência de altos teores de $\mathrm{Mg}, \mathrm{K}$ e $\mathrm{N}$ (Ferguson \& Watkins, 1989; Pavicic et al., 2004; Argenta \& Suzuki, 1994; Nachtigall \& Freire, 1998; Amarante et al., 2006a; Amarante et al., 2006b). A deficiência de Ca pode reduzir a integridade de estrutura da parede celular e comprometer a permeabilidade seletiva das membranas celulares, resultando em injúria e necrose dos tecidos (Chardonnet et al., 2003; Saure, 2005). Além disto, o Ca tem importante papel regulatório no metabolismo celular (Saftner et al., 1998; Taiz \& Zeiger, 2006).

O risco de ocorrência de "bitter pit" é normalmente avaliado por meio da análise mineral (teores de $\mathrm{Ca}, \mathrm{N}, \mathrm{K}$ e $\mathrm{Mg}$ ) dos frutos. Todavia, estas análises são caras, relativamente demoradas, requerem equipamentos especializados e, muitas vezes, apresentam baixa capacidade de predição do risco de ocorrência da desordem. Além disso, devido à grande variabilidade entre frutos em um mesmo talhão de pomar, é necessário maior número de amostras por unidade de área do pomar para ter boa predição do risco de "bitter pit" (Ferguson \& Watkins, 1989). Isto tem estimulado o estudo de métodos alternativos que permitam, de forma rápida, prática e econômica, a predição do risco de ocorrência desse distúrbio em maçãs.

Burmeister \& Dilley (1991 e 1994) reportaram a indução de sintomas de "bitter pit" em maçãs infiltradas com solução de $\mathrm{Mg}$. Segundo esses autores, frutos com baixas concentrações de $\mathrm{Ca}$ foram suscetíveis ao desenvolvimento de "bitter pit" induzido por infiltração com Mg. Em adição, o desenvolvimento de "bitter pit" por infiltração com $\mathrm{Mg}$ foi reduzido quando concentrações crescentes de cloreto de cálcio foram incluídas no meio de infiltração. Diversos autores observaram boa correlação entre "bitter pit" induzido por infiltração com $\mathrm{Mg}$ e a incidência de "bitter pit" após armazenamento refrigerado (Burmeister \& Dilley, 1994; Retamales et al., 2000; Amarante et al., 2005). Através deste método, os frutos são colhidos cerca de 20 dias antes da colheita comercial, infiltrados a vácuo em uma solução de $\mathrm{MgCl}_{2}$ e avaliados quanto à incidência e severidade de sintomas tipo "bitter pit" após serem deixados durante 10-14 dias em temperatura ambiente (Burmeister \& Dilley, 1991; Burmeister \& Dilley, 1994; Retamales et al., 2000; Amarante et al., 2005). A infiltração com $\mathrm{Mg}$ pode permitir rápida e eficiente identificação de frutos quanto à suscetibilidade ao "bitter pit" no momento da colheita comercial.

O objetivo deste trabalho foi avaliar a viabilidade prática da infiltração de maçãs 'Gala' com $\mathrm{Mg}$, visando a identificar em pré-colheita, o risco de ocorrência de "bitter pit" durante o armazenamento refrigerado dos frutos. Adicionalmente, procurouse identificar os atributos minerais que melhor discriminam diferenças entre pomares comerciais quanto ao risco de "bitter pit".

\section{MATERIAL E MÉTODOS}

O experimento foi conduzido com frutos coletados em pomares comerciais localizados no município de Fraiburgo-SC, na safra de 2002/2003. Foram utilizados 50 talhões para a colheita dos frutos, com área aproximada de 1 ha cada.

Cerca de 20 dias antes do início da colheita comercial, foram coletadas amostras de 25 frutos em cada talhão, para a infiltração com $\mathrm{Mg}$. Os frutos, de tamanho uniforme (100-120g), foram colhidos de forma aleatória, no terço médio de todo o perímetro da copa das plantas. Foi colhido um fruto por planta, escolhida aleatoriamente, de maneira a representar todo o talhão. Após a colheita, os frutos foram deixados a $20 \pm 4^{\circ} \mathrm{C} / 60-70 \%$ UR durante 24 horas. A seguir, foram submersos em solução de $\mathrm{MgCl}_{2} 0,1$ mol.L ${ }^{-1}$, contendo 0,3 mol.L-1 de sorbitol (como agente osmótico) e o espalhante adesivo Silwet L-77 AG $(0,05 \%)$, e infiltrados a vácuo $(150 \mathrm{~mm} \mathrm{Hg})$ durante dois minutos. Os frutos infiltrados foram avaliados quanto à incidência (\%) e severidade (manchas/fruto) de manchas tipo "bitter pit" induzidas pelo $\mathrm{Mg}$, após um período de 10 dias a $20 \pm 4^{\circ} \mathrm{C} / 60-70 \%$ UR.

$\mathrm{Na}$ maturação comercial, foram coletadas amostras de 120 frutos / talhão. Cem frutos foram armazenados em câmara fria convencional durante 
quatro meses $\left(0 \pm 0,5^{\circ} \mathrm{C}\right.$ e $90-95 \%$ UR), e avaliados quanto à incidência (\%) e severidade (manchas / fruto) de "bitter pit", cinco dias após remoção da câmara fria. Vinte frutos foram utilizados para a quantificação dos teores de $\mathrm{Ca}, \mathrm{Mg}, \mathrm{K}$ e N.

Para a análise mineral, os frutos foram previamente lavados com água destilada. Posteriormente, retirou-se uma fatia longitudinal (menos pedúnculo e semente), contendo tecidos de casca e polpa. O tecido fresco foi digerido a $350^{\circ} \mathrm{C}$, usando-se uma mistura de ácido sulfúrico concentrado $\left(\mathrm{H}_{2} \mathrm{SO}_{4}\right)$ e água oxigenada $\left(\mathrm{H}_{2} \mathrm{O}_{2}, 30\right.$ volumes). $\mathrm{O} \mathrm{N}$ foi determinado pelo método semimicro-Kjeldahl, como descrito por Tedesco et al. (1995), e os demais nutrientes (K, $\mathrm{Ca}$ e $\mathrm{Mg}$ ) foram determinados por espectrofometria de emissão induzida por plasma. Os teores dos diferentes elementos foram expressos em mg. $\mathrm{kg}^{-1} \mathrm{de}$ matéria fresca.

Os dados obtidos foram submetidos à análise estatística, utilizando-se do programa SAS (SAS Institute, 2002). Foram realizadas análises de correlação, para os valores de incidência e severidade de "bitter pit", entre frutos infiltrados com Mg e frutos armazenados em câmara fria, oriundos de diferentes talhões. Os atributos minerais de frutos (infiltrados com $\mathrm{Mg}$ e frutos armazenados em câmara fria), originados de talhões com elevada e com baixa incidência e severidade de "bitter pit", foram submetidos à análise multivariada (análise canônica discriminante: ACD). Isto foi realizado, visando a identificar os atributos minerais que melhor permitem discriminar diferenças entre talhões quanto à incidência e severidade ao "bitter pit" em maçãs 'Gala', bem como a verificar a consistência destes resultados em frutos infiltrados com $\mathrm{Mg}$ e em frutos armazenados em câmara fria. $\mathrm{O}$ parâmetro adotado para avaliação do efeito de separação, gerado pelos atributos minerais, entre os níveis de incidência e severidade estudados, foi o coeficiente da taxa de discriminação paralela (TDP), obtido através do produto entre valores dos coeficientes canônicos padronizados (CCP) e os valores dos coeficientes de correlação canônica (r), conforme descrito por Amarante et al. (2006a).

\section{RESULTADO E DISCUSSÃO}

A incidência de "bitter pit" em frutos armazenados em câmara fria apresentou correlação linear altamente significativa com a incidência desse distúrbio em frutos infiltrados com $\mathrm{Mg}\left(\mathrm{r}^{2}=0,69\right.$; $\mathrm{p}<0,001$ ) (Figura 1). Isto ocorreu em maçãs coletadas em um grande número de talhões, que podem apresentar diferenças quanto à nutrição e manejo das plantas. Mesmo tendo havido uma superestimativa da incidência do distúrbio nos frutos infiltrados em relação aos frutos armazenados (Figura 1), esse método mostrou-se eficiente na capacidade de identificar lotes com a presença de "bitter pit", apesar de não ter sido eficiente para avaliar a severidade do distúrbio entre os dois grupos de frutos (dados não apresentados). Portanto, a infiltração com $\mathrm{Mg}$ é capaz de induzir o aparecimento de "bitter pit" nos tecidos predisponentes à manifestação do distúrbio, principalmente na região do cálice, onde a concentração de Ca é menor (Lewis \& Martin, 1973; Ferguson \& Watkins, 1983), da mesma forma como observado em frutos que foram simplesmente armazenados em câmara fria.

Após o aparecimento de "bitter pit" nos frutos pela infiltração com $\mathrm{Mg}$, os talhões foram divididos em dois grupos, apresentando (incidência de 4 a 27\% e severidade média de 0,1 a 5,6 manchas / fruto) ou não "bitter pit". Para avaliações feitas após armazenamento em câmara fria, os talhões foram classificados como tendo incidência baixa (0-5\%) e alta (7-18\%), e severidade média baixa (0-0,1 manchas / fruto) e alta (de 0,1 a 5,6 manchas / fruto) de "bitter pit". Os atributos relacionados com a composição mineral dos frutos amostrados na colheita, correspondentes a talhões pertencentes a um mesmo grupo de incidência ou severidade de "bitter pit", para frutos infiltrados com $\mathrm{Mg}$ ou armazenados em câmara fria, foram submetidos a ACD. Na ACD, foram considerados os teores de $\mathrm{Ca}$ e as relações deste elemento com os demais $(\mathrm{Mg}, \mathrm{K}$ e $\mathrm{N})$, já que apresentam clara relação com a ocorrência de "bitter pit" em maçãs (Ferguson \& Watkins, 1989; Amarante et al., 2006a; Amarante et al., 2006b). Para os elementos individuais, apenas os teores de $\mathrm{N}$ foram incluídos na ACD, por apresentarem diferença altamente significativa na análise univariada, entre grupos com diferentes níveis de incidência ou severidade de "bitter pit", em frutos infiltrados com $\mathrm{Mg}$ e em frutos armazenados em câmara fria (dados não apresentados).

$\mathrm{O}$ teor de $\mathrm{N}$ e a relação $\mathrm{N} / \mathrm{Ca}$ nos frutos apresentaram maiores valores de TDP, sendo os principais responsáveis pela discriminação entre grupos de incidência ou severidade de "bitter pit", em frutos infiltrados com $\mathrm{Mg}$ e em frutos armazenados em câmara fria (Tabela 1). Em frutos infiltrados com $\mathrm{Mg}$, o teor de $\mathrm{N}$ foi mais importante para discriminar lotes quanto à incidência, enquanto a relação $\mathrm{N} / \mathrm{Ca}$ foi mais importante para discriminar lotes quanto à severidade de "bitter pit". Em frutos armazenados em câmara fria, a relação $\mathrm{N} / \mathrm{Ca}$ foi o atributo mais importante para discriminar lotes quanto ao grau de incidência e severidade de "bitter pit", sendo mais 
importante do que o teor de Ca.

A relação $\mathrm{Mg} / \mathrm{Ca}$ apresentou valores elevados de TDP (Tabela 1). Todavia, isto não mostrou relação com a incidência de "bitter pit", já que lotes de frutos com alta incidência e severidade apresentaram menores valores da relação $\mathrm{Mg} / \mathrm{Ca}$ (dados não apresentados). A manifestação de "bitter pit" em maçãs normalmente se verifica em frutos com alta relação $\mathrm{Mg} / \mathrm{Ca}$ (Ferguson \& Watkins, 1989; Argenta \& Suzuki, 1994; Nachtigall \& Freire, 1998; Amarante et al., 2006b). Portanto, talhões com maior ocorrência de "bitter pit" apresentaram, por algum fator relacionado à nutrição e/ou ao manejo adotados, menores teores de $\mathrm{Mg}$ nos frutos, os quais não apresentaram relação com o desenvolvimento do distúrbio.

A incidência e a severidade de "bitter pit" foram mais elevadas nos frutos com baixos teores de $\mathrm{Ca}$ e altos teores de $\mathrm{N}$, tanto nos infiltrados com $\mathrm{Mg}$ como naqueles armazenados sob refrigeração durante quatro meses (Figura 2).

Talhões cujos frutos tinham teores de $\mathrm{N}$ inferiores a 400 mg. $\mathrm{kg}^{-1}$, mesmo apresentando ampla faixa de teores de $\mathrm{Ca}$ (variando de 18 a $42 \mathrm{mg} . \mathrm{kg}^{-1}$ ), apresentaram níveis de incidência e severidade de "bitter pit" próximos de zero, independentemente do grupo de origem dos frutos (infiltrados com $\mathrm{Mg}$ ou armazenados em câmara fria) (Figura 2). Apenas um talhão apresentou frutos com teor de Ca superior a $42 \mathrm{mg} \cdot \mathrm{kg}^{-1}$, e mesmo com teor de $\mathrm{N}$ próximo de 600 $\mathrm{mg} . \mathrm{kg}^{-1}$, esses frutos apresentaram baixa incidência e baixa severidade de "bitter pit".

Em talhões cujos frutos tinham teores de $\mathrm{N}$ entre 400 e 500 mg. $\mathrm{kg}^{-1}$ e teores de Ca entre 18 e 42 mg. $\mathrm{kg}^{-1}$, a incidência e a severidade de "bitter pit" foram baixas (Figura 2). Apenas um talhão, com frutos apresentando $460 \mathrm{mg} \cdot \mathrm{kg}^{-1}$ de $\mathrm{N}$ e $28 \mathrm{mg} . \mathrm{kg}^{-1}$ de $\mathrm{Ca}$, apresentou elevada ocorrência de "bitter pit". Em talhões cujos frutos tinham teores de $\mathrm{Ca}$ entre 36 e $42 \mathrm{mg} \cdot \mathrm{kg}^{-1}$ e teores de $\mathrm{N}$ entre 500 e 720 mg. $\mathrm{kg}^{-1}$, a incidência e a severidade de "bitter pit" também foram baixas, independentemente do lote de frutos (infiltrados com $\mathrm{Mg}$ ou armazenados em câmara fria) (Figura 2).

Níveis moderados a elevados de incidência e severidade de "bitter pit" foram observados em talhões nos quais, concomitantemente, os teores de Ca foram menores do que $36 \mathrm{mg} \cdot \mathrm{kg}^{-1}$, e os teores de $\mathrm{N}$ foram maiores do que $500 \mathrm{mg} \cdot \mathrm{kg}^{-1}$, tanto em frutos infiltrados com $\mathrm{Mg}$ como em frutos armazenados em câmara fria (Figura 2).

Os resultados obtidos, relacionando teores de Ca e de N e risco de "bitter pit", observados em frutos infiltrados com $\mathrm{Mg}$ e em frutos armazenados em câmara fria (Figura 2), são apresentados na forma de diagrama, na Figura 3.

A grande maioria dos autores relaciona a ocorrência de "bitter pit" em maçãs com os baixos teores de $\mathrm{Ca}$ e elevadas relações $\mathrm{K} / \mathrm{Ca}, \mathrm{Mg} / \mathrm{Ca}$ e $(\mathrm{K}+\mathrm{Mg}) / \mathrm{Ca}$ nos frutos (Ferguson \& Watkins, 1989; Argenta \& Suzuki, 1994; Nachtigall \& Freire, 1998; Amarante et al., 2006a; Amarante et al., 2006b). Todavia, o suprimento de $\mathrm{N}$ em excesso aumenta o rico de "bitter pit" em macieiras, pois favorece o crescimento vegetativo, aumentando assim o suprimento de $\mathrm{Ca}$ às folhas em detrimento dos frutos (Ferguson \& Watkins, 1989). Em macieiras 'Fuji', o aumento nas doses de $\mathrm{N}$ (de 28,4 a 170,5 kg.ha-1) elevou os teores de $\mathrm{N}$ nas folhas e nos frutos, e reduziu a firmeza de polpa e aumentou a incidência de "bitter pit" e de escaldadura nos frutos (Raese \& Drake, 1997).

Em macieiras cultivadas na região Sul do Brasil, em solos com médio a alto teor de matéria orgânica, o suprimento de $\mathrm{N}$ nativo do solo e dos resíduos culturais em decomposição tem-se mostrado suficiente para a manutenção de produtividades e vigor desejáveis das plantas (Ernani \& Dias, 1999; Ernani et al., 2000). A região de Fraiburgo-SC, não apresenta condições adequadas de requerimento em frio para o cultivo de macieira no Sul do Brasil. Isto normalmente propicia a rápida mineralização de $\mathrm{N}$ da matéria orgânica do solo e aumenta a atividade radicular de absorção de nutrientes, especialmente durante a primavera e o verão. Desta forma, o aumento no suprimento de $\mathrm{N}$ às plantas, através de adubação química ou orgânica, pode aumentar a relação $\mathrm{N} / \mathrm{Ca}$ nos frutos, elevando o risco de "bitter pit". Portanto, em maçãs 'Gala' cultivadas nestas condições, cuidados especiais devem ser tomados visando a evitar o excesso de $\mathrm{N}$, em associação a medidas de manejo e práticas culturais que reduzem o risco de ocorrência de "bitter pit".

A correlação significativa para a incidência de "bitter pit", entre os frutos infiltrados com Mg e os armazenados em câmara fria, mostra que a infiltração com Mg permite avaliar em pré-colheita o risco pós-colheita do distúrbio. Em adição a isto, houve correspondência quanto aos níveis críticos de $\mathrm{Ca}$ e $\mathrm{N}$ nos frutos para a ocorrência de "bitter pit", tanto em frutos infiltrados com $\mathrm{Mg}$ como em frutos armazenados sob refrigeração durante quatro meses. Desta forma, frutos de talhões ou pomares com elevado risco de ocorrência de "bitter pit", identificados através do método de infiltração com $\mathrm{Mg}$, podem ser segregados na colheita e destinados para a imediata comercialização ou armazenamento refrigerado de curta duração. 
TABELA 1- Coeficiente da taxa de discriminação paralela (TDP) para a função canônica discriminante 1 $\left.(\mathrm{FCD})_{1}\right)$, referente às análises dos elementos minerais, e suas relações com $\mathrm{Ca}$, em maçãs 'Gala' provenientes de talhões com baixa e alta incidência e severidade de "bitter pit", avaliados em frutos infiltrados com $\mathrm{Mg}$ e em frutos armazenados durante quatro meses em câmara fria (Fraiburgo-SC).

\begin{tabular}{lcc}
\hline Atributos avaliados & Incidência & Severidade \\
\hline & Frutos & infiltrados com $\mathbf{M g}$ \\
$\mathrm{Ca}$ & $-0,0041$ & 0,0195 \\
$\mathrm{~N}$ & 0,6220 & 0,1094 \\
$\mathrm{~N} / \mathrm{Ca}$ & 0,2043 & 0,5137 \\
$\mathrm{~K} / \mathrm{Ca}$ & $-0,0508$ & $-0,0909$ \\
$\mathrm{Mg} / \mathrm{Ca}$ & 0,2286 & 0,4483 \\
$(\mathrm{~K}+\mathrm{Mg}) / \mathrm{Ca}$ & 0,0000 & 0,0000 \\
$(\mathrm{~K}+\mathrm{Mg}+\mathrm{N}) / \mathrm{Ca}$ & 0,0000 & 0,0000 \\
\hline & Frutos armazenados em câmara fria \\
$\mathrm{Ca}$ & 0,3126 & 0,4363 \\
$\mathrm{~N}$ & $-0,7137$ & $-0,5983$ \\
$\mathrm{~N} / \mathrm{Ca}$ & 0,9422 & 0,8386 \\
$\mathrm{~K} / \mathrm{Ca}$ & $-0,2076$ & $-0,1051$ \\
$\mathrm{Mg} / \mathrm{Ca}$ & 0,6666 & 0,4286 \\
$(\mathrm{~K}+\mathrm{Mg}) / \mathrm{Ca}$ & 0,0000 & 0,0000 \\
$(\mathrm{~K}+\mathrm{Mg}+\mathrm{N}) / \mathrm{Ca}$ & 0,0000 & 0,0000 \\
\hline
\end{tabular}

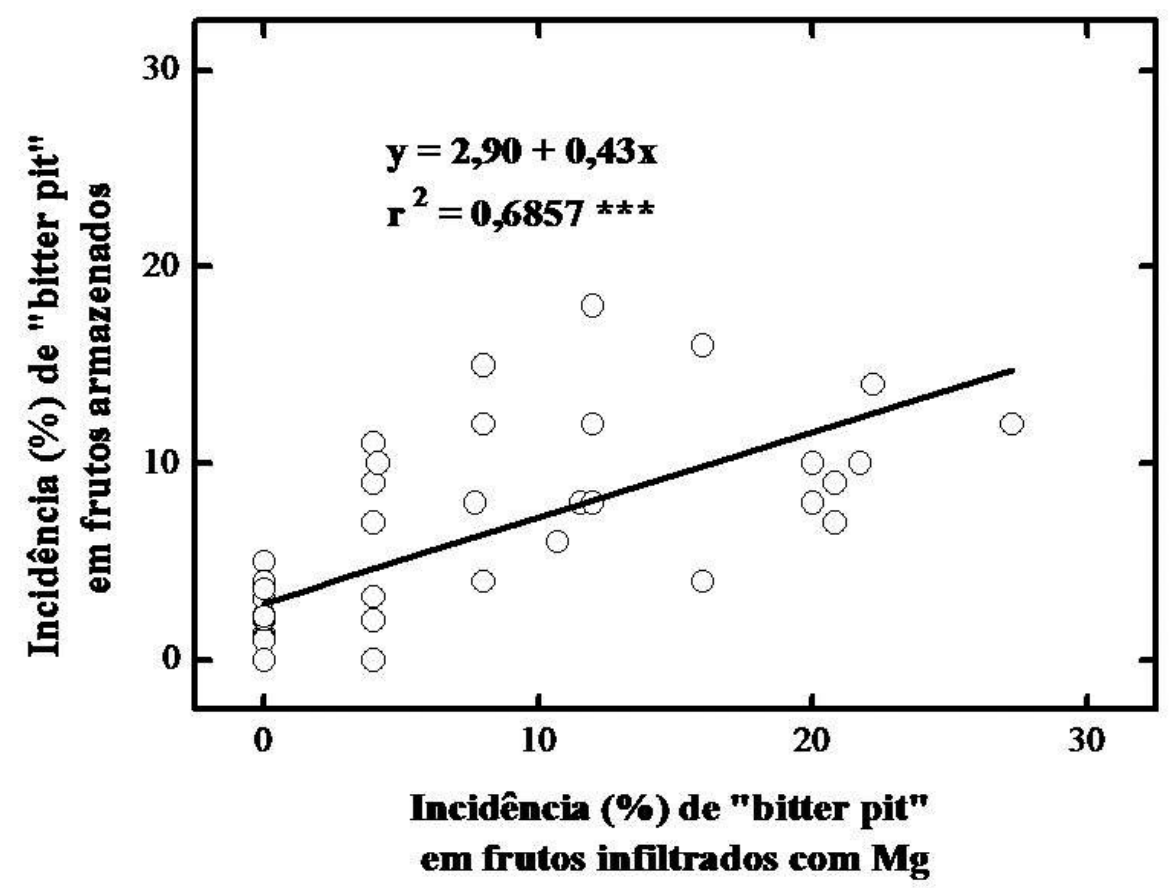

FIGURA 1 - Relação entre incidência de "bitter pit", avaliada em frutos infiltrados com Mg e em frutos armazenados em câmara fria, em maçãs 'Gala' colhidas em 50 talhões de pomares comerciais, no município de Fraiburgo-SC. 

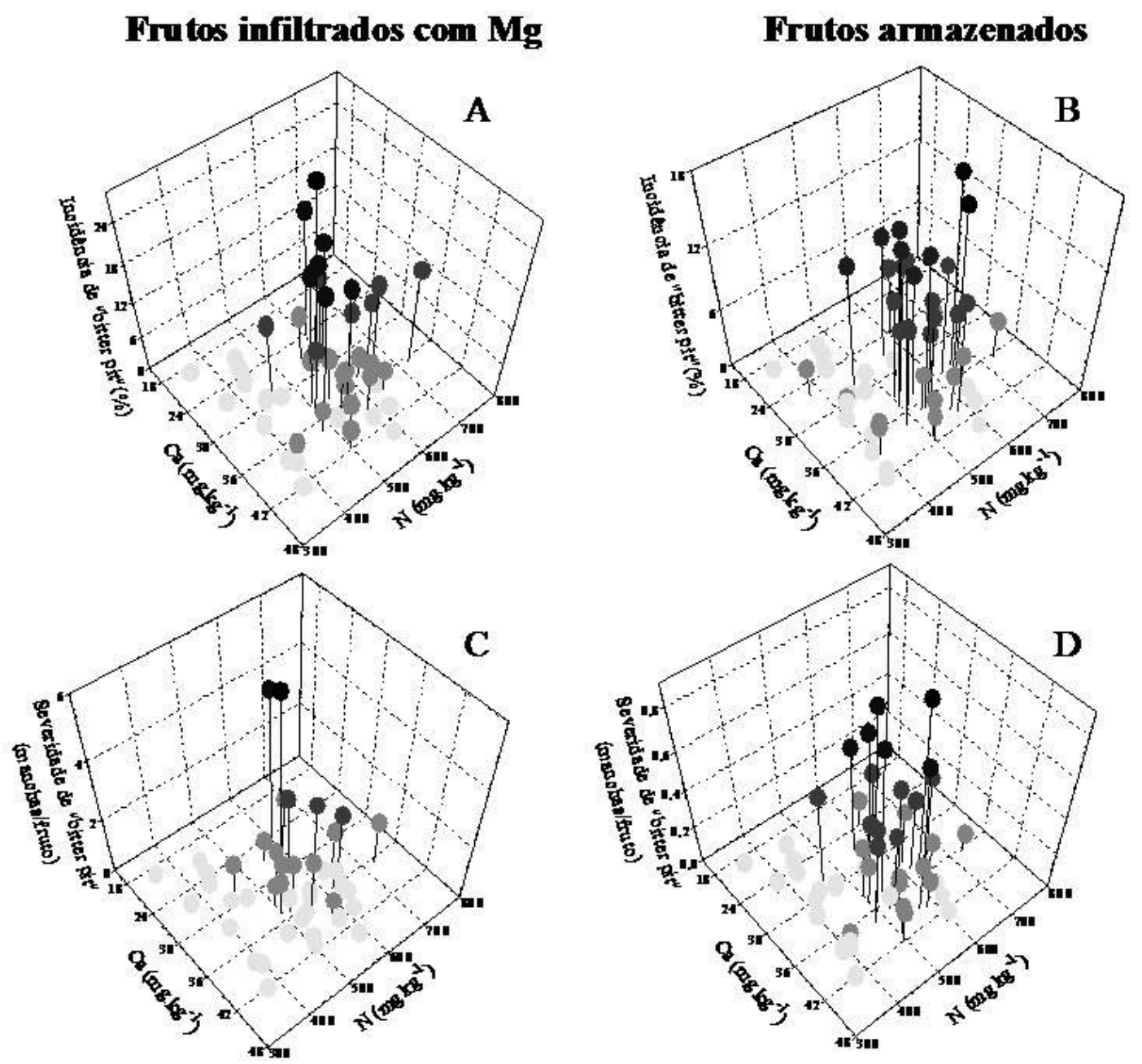

FIGURA 2 - Incidência (A e B) e severidade (C e D) “bitter pit” em maçãs 'Gala', após infiltração com $\mathrm{Mg}$ (gráficos à esquerda) ou quatro meses de armazenamento em câmara fria convencional (gráficos à direita), em função dos teores de $\mathrm{Ca}$ e $\mathrm{N}$ nos frutos (Fraiburgo-SC).

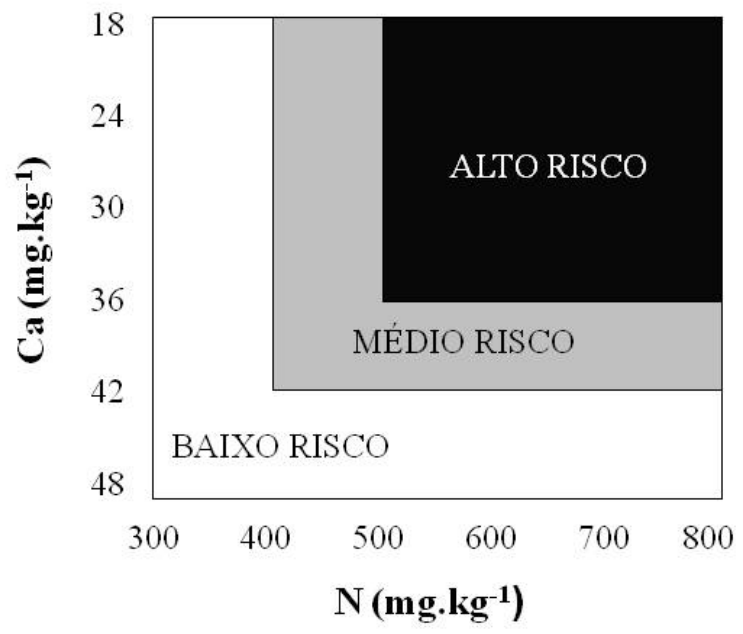

FIGURA 3 - Diagrama da relação entre teores de Ca e N nos frutos e o risco pós-colheita de ocorrência de "bitter pit" em maçãs 'Gala'. 


\section{CONCLUSÕES}

1-Houve correlação significativa entre a incidência de "bitter pit" avaliada em frutos infiltrados com $\mathrm{Mg}$ e em frutos armazenados em câmara fria.

2-Houve correspondência quanto aos níveis críticos de $\mathrm{Ca}\left(<36 \mathrm{mg} \cdot \mathrm{kg}^{-1}\right)$ e $\mathrm{N}\left(>500 \mathrm{mg} \cdot \mathrm{kg}^{-1}\right)$ em maçãs 'Gala', relacionados ao elevado risco de "bitter pit", em frutos infiltrados com $\mathrm{Mg}$ e em frutos armazenados sob refrigeração durante quatro meses.

\section{AGRADECIMENTOS}

Os autores agradecem ao Conselho Nacional de Desenvolvimento Científico e Tecnológico $(\mathrm{CNPq})$, pelo apoio financeiro a este projeto.

\section{REFERÊNCIAS}

AMARANTE, C.V.T. do; CHAVES, D.V.; ERNANI, P.R. Análise multivariada de atributos nutricionais associados ao "bitter pit” em maçãs 'Gala'. Pesquisa Agropecuária Brasileira, Brasília, v.41, n.5, p.841846, 2006a.

AMARANTE, C.V.T. do; CHAVES, D.V.; ERNANI, P.R. Composição mineral e severidade de "bitter pit" em maçãs 'Catarina'. Revista Brasileira de Fruticultura, Jaboticabal, v.28, n.1, p.51-54, 2006 b.

AMARANTE, C.V.T. do; ERNANI, P.R.; CHAVES, D.V. Fruit infiltration with Magnesium is a feasible way to predict bitter pit susceptibility in 'Gala' apples grown in Southern Brazil. Acta Horticulturae, Leuven, n.682, p.1271-1274, 2005.

ARGENTA, L.C.; SUZUKI, A. Relação entre teores minerais e frequência de bitter pit em maçã cv. Gala no Brasil. Revista Brasileira de Fruticultura, Jaboticabal, v.16, n.1, p.267-277, 1994.

BURMEISTER, D.M.; DILLEY, D.R. Correlation of bitter pit on Northern Spy apples with bitter pitlike symptoms induced by $\mathrm{Mg}^{+2}$ salt infiltration. Postharvest Biology and Technology, Amsterdam, v.4, n.4, p.301-308, 1994.

BURMEISTER, D.M.; DILLEY, D.R. Induction of bitter pit-like symptoms on apples by infiltration with $\mathrm{Mg}^{+2}$ is attenuated by $\mathrm{Ca}^{+2}$. Postharvest Biology and Technology, Amsterdam, v.1, n.1, p.11-17, 1991.
CHARDONNET, C.O.; CHARRON, C.S.; SAMS, C.E.; CONWAY, W.S. Chemical changes in the cortical tissue and cell walls of calciuminfiltrated 'Golden Delicious' apples during storage. Postharvest Biology and Technology, Amsterdam, v.28, n.1, p.97-111, 2003.

DRAZĚTA, L.; LANG, A.; CAPPELLINI, C.; HALL, A.J.; VOLZ, R.K.; JAMESON, P.E. Vessel differentiation in the pedicel of apple and the effects of auxin transport inhibition. Physiologia Plantarum, Copenhague, v.120, n.1, p.162-170, 2004.

ERNANI, P.R.; DIAS, J. Soil nitrogen application in the spring did not increase apple yield. Ciência Rural, Santa Maria, v.29, n.4, p.645-649, 1999.

ERNANI, P.R.; DIAS, J.; BORGES, M. A aplicação de nitrogênio ao solo em diferentes estádios não afetou o rendimento de frutos de cultivares de macieira. Ciência Rural, Santa Maria, v.30, n.2, p.223-227, 2000.

FERGUSON, I.B.; WATKINS, C.B. Bitter-pit in apple fruit. Horticultural Reviews, New York, v.11, p.289-355, 1989.

FERGUSON, I.B.; WATKINS, C.B. Cation distribution and balance in apple fruit in relation to calcium treatments for bitter pit. Scientia Horticulturae, Amsterdam, v.19, n.3/4, p.301-310, 1983.

LEWIS, T.L.; MARTIN, D. Longitudinal distribution of applied calcium, and of naturally occurring calcium, magnesium, and potassium in Merton apple fruits. Australian Journal of Agricultural Research, Collingwood, v.24, n.3, p.363-371, 1973.

NACHTIGALL, G.R.; FREIRE, C.J.S. Previsão da incidência de "bitter pit" em maçãs através dos teores de cálcio em folhas e frutos. Revista Brasileira de Fruticultura, Jaboticabal, v.20, n.2, p.158-166, 1998.

PAVICIC, N.; JEMRIC, T.; KURTANJEK, Z.; COSIC, T.; PAVLOVIC, I.; BLASKOVIC, D. Relationship between water-soluble $\mathrm{Ca}$ and other elements and bitter pit occurrence in 'Idared' apples: a multivariate approach. Annals of Applied Biology, Londres, v.145, n.2, p.193-196, 2004. 
RAESE, J.T.; DRAKE, S.R. Nitrogen fertilization and elemental composition affects fruit quality of 'Fuji' apples. Journal of Plant Nutrition, Londres, v.20, n.12, p.1797-1809, 1997.

RETAMALES, J.B.; VALDES, C.; DILLEY, D.R.; LEÓN, L.; LEPE, V.P. Bitter pit prediction in apples through $\mathrm{Mg}$ infiltration. Acta Horticulturae, Leuven, n.512, p.169-179, 2000.

SAFTNER, R.A.; CONWAY, W.S.; SAMS, C.E. Effects of postharvest calcium and fruit coating treatments on postharvest life, quality maintenance, and fruit-surface injury in 'Golden Delicious' apples. Journal of the American Society for Horticultural Science, Alexandria, v.123, n.2, p.294-298, 1998.
SAS INSTITUTE. Getting started with the SAS learning edition. Cary: SAS, 2002. 200p.

SAURE, M.C. Calcium translocation to fleshy fruit: its mechanism and endogenous control. Scientia Horticulturae, Amsterdam, v.105, n.1, p.65-89, 2005.

TAIZ, L.; ZEIGER, E. Plant physiology. 4.ed. Sunderland: Sinauer Associates, 2006. 705p.

TEDESCO, M.J.; GIANELLO, C.; BISSANI, C.A.; BOHNEN, H.; VOLKWEISS, S.J. Análise do solo, planta e outros materiais. 2.ed. Porto Alegre: Departamento de Solos, UFRGS, 1995. 174p. (Boletim Técnico de Solos, 5). 